\title{
CUERPO Y OÍDO PENSANTE: MÉTODO DE FORMACIÓN MUSICAL PARA PERSONAS CON UN NIVEL APTITUDINAL DISCIPLINARMENTE NO APTO
}

\author{
Miguel Fernando Montes Toro'
}

\section{Resumen}

Por lo general, son los conservatorios y las escuelas de música las instituciones encargadas de los procesos de formación académica inicial y profesional en las líneas instrumental, teórica y técnica. Es requisito para acceder a este sistema de formación musical académica el contar con aptitudes personales evaluadas por medio de instrumentos específicos. Los instrumentos utilizados para la selección de ingreso de candidatos siguen las pautas estructurales del currículo musical centrado en la evaluación de competencias tonales, rítmicas e instrumentales, el cual excluye a quienes, dentro de esta escala, se consideran arrítmicos, atonales y de bajo desarrollo motor, sin que se les ofrezcan posibilidades de formación musical acorde con sus aptitudes "disfunsivas". La exclusión generada por las pautas de la formación académica musical, centrada en los talentosos, y su incapacidad para incluir a los que no han desarrollado su talento musical, amerita buscar soluciones acordes con las condiciones específicas de este segundo grupo con el propósito de desarrollar una creación musical según sus posibilidades rítmicas, melódicas y compositivas. Esta fractura disciplinar y social, tanto de costumbres musicales como de producción teórica, sugiere, en este caso, analizar las categorías principales que constituyen la teoría de la inteligencia musical propuesta por Gardner con el propósito de ampliar el espectro tradicional del aprendizaje y la práctica musical hacia niveles de comprensión que la hagan asequible a otros grupos humanos interesados en ella.

\section{Palabras claves}

Creación musical, partitura textual, partitura musical, partitura corporal, aptitud, C.O.P. (cuerpo y oído pensante).

Docente investigador de la Facultad de Cultura Física de la Universidad Santo Tomás. Proyecto presentado para optar al título de Doctorado en Educación de la Universidad de Costa Rica. San José de Costa Rica. Email:miguelmontes@correo.usta.edu.co 


\begin{abstract}
In general, the conservatories and the music schools are the institutions in charge of the initial and professional academic formation in instruments, theory and techniques. One of the requirements to access this systems is to have personal aptitudes evaluated with specific tools. These tools used to select the candidates follow some structural patterns of the musical syllabus focused on the evaluation of instrumental, rhyme, and tune competence which exclude those, who in this scale, are considered no fit with a low development without offering them other possibilities of musical education according to their aptitudes. The exclusion generated by the norms of academic studies, focused on talented people and its incapacity to include those from the second group, in order to develop a musical creation according to their possibilities. This social and disciplinary fracture of musical customs as well of the theoretical production, suggests in this case, the need to analyze the principal categories that constitute the theory of the musical intelligence proposed by Gardner in order to broaden the traditional learning scheme and the musical practice toward levels of understanding that make it affordable by other human groups interested in it.
\end{abstract}

\title{
Index terms
}

Musical creation, musical score, corporal score, aptitud, T.B.E. (thinking body and ear).

\section{Introducción}

Las tradiciones académicas en el campo de la investigación en educación musical se han orientado a la comprensión de los fenómenos propios de la música a partir de los patrones y de los modelos teóricos que esta presenta, preconcebidos en hipótesis que han determinado la marcha en el terreno de la investigación en educación musical y por análisis positivistas que atienden necesidades primarias de las técnicas, la interpretación, la gramática y la estructura tonal.

Las tradiciones desde las que se pretende abordar la construcción del método están enmarcadas dentro de la epistemología de los métodos cualitativos, con el fin de comprender los fenómenos y las categorías que integran la estructura "cuerpo y oído pensante".

Los reportes de investigación seleccionados para la etapa preliminar de lectura y de revisión bibliográfica se han enfocado al análisis de las premisas teóricas y metodológicas que plantea cada uno de ellos, con el fin de seleccionar los documentos que más puedan aportar a la consolidación del método de formación musical Cuerpo y oído pensante. La estructura de los documentos revisados está dividida en conferencias, plenarias, informes de investigación y ponencias, desarrolladas por profesionales de la música en el nivel de maestría y doctorado. Estos documentos son publicaciones seriadas, indexadas y electrónicas.

En América Latina la poca producción investigativa en este campo, sugiere que la consolidación de centros de investigación y de instituciones dedicadas a esta labor son realmente escasas. Los centros de investigación en artes musicales de América Latina se formalizan en los centros de educación superior, con el desarrollo de proyectos de grado para optar al título de maestro en música o de pedagogo musical en prácticas instrumentales, técnicas y mecánicas, que antes que 
generar procesos evolutivos de la investigación misma, hacen más elemental su práctica y trascendencia.

La investigación en educación musical surge en los países europeos y de allí se da a conocer en el mundo a partir de las tradiciones generadas por métodos convencionales de la enseñanza musical, hasta los nuevos posicionamientos conceptuales de los teóricos actuales, que continúan socavando en los métodos antiguos y en la estructura tradicional, como únicas posibilidades de trascender y argumentar la práctica musical.

Estos posicionamientos teóricos, basados en la repetición, la técnica y la instrumentalización musical argumentan mucho más la necesidad de formalizar un método de educación musical, propio, tanto para quienes han estado excluidos de esta expresión estética y artística por considerársele sin aptitud, como para los que disciplinarmente se consideran talentosos.

Un estado del arte de la investigación en educación musical deja percibir claramente las diferentes perspectivas epistemológicas más usuales en dicho campo. Igualmente, permite identificar referentes teóricos claros que en algunos casos se oponen entre ellos y dejan entrever los intereses de la investigación en educación musical. Además, permite identificar los diferentes paradigmas de investigación en los cuales se desarrolla actualmente la investigación en educación musical, para dejar en evidencia la posibilidad de abordar este campo desde una posición cualitativa, cuantitativa o emergente.

Los reportes de investigación se clasificaron de acuerdo con las tendencias epistemológicas en las que cada uno de ellos se inserta, y, en la mayoría de los casos, la posibilidad más cercana al ejercicio investigativo se proyecta, por un lado, desde la corriente positivista, para esperar conseguir resultados que identifiquen inexactitudes en el estudio y aprendizaje de la música, además de la consecución de un estado de la cuestión a partir del censo y de la recopilación de datos estadísticos, como número de estudiantes, tipos de instrumentos, corrientes pedagógicas y métodos de enseñanza apren- dizaje; por otra parte, también se observa una tendencia de trabajo investigativo cualitativo, que se proyecta frente al tema de la educación musical, y de la investigación en educación musical, como una posibilidad de abrir puertas a nuevas experiencias y tendencias conceptuales de la enseñabilidad y educabilidad de la música en el contexto básico y superior.

Estos referentes contextualizan el sistema problemático del método, en relación con la multiplicidad de corrientes investigativas que convergen en la investigación educativa actual en el campo de la educación musical. Esto presenta una visión mucho más amplia de las posibilidades de búsqueda y análisis que se pueden tener en cuenta para consolidar un método que convoque y acierte con las necesidades de la educación musical.

Las diferentes discusiones presentadas en los reportes de investigación permiten establecer tanto las controversias más actuales en el campo de la investigación en educación musical, como las tendencias. Los tipos de investigación que convergen en la actualidad permiten romper con esa tradición excluyente y purista del ejercicio musical desde la práctica que se genera en los conservatorios, en la etapa de formación profesional, hasta las metodologías que se aplican en las instituciones educativas del nivel básico y de formación preliminar; esto constituye un paso fundamental que permite avizorar un cambio significativo en las prácticas académicas y de formación musical, aplicada a los contextos y a las necesidades propias de los sujetos.

Los ejes centrales de interés de los reportes de investigación sistematizados para este proyecto establecen aportes significativos para concretar los puntos álgidos de la discusión que se genera en torno a la educación musical; unos continúan anquilosados en la técnica y en la formación instrumental como única posibilidad de avanzar en la producción del conocimiento musical, y otros permiten ver claramente que esa etapa positivista de la música requiere de ajustes y cambios concretos que puedan hacer del conocimiento musical algo útil y propio a las condiciones aptitudinales específicas de los sujetos. 
Las tradiciones musicales provenientes de corrientes en su mayoría anglosajonas se han expandido por el mundo como la manifestación más pura de la música, que hibrída costumbres, culturas, tradiciones, sensibilidades, estratos, y asociaciones tribales de las más recónditas y alejadas. El estudio de la música en los conservatorios, en su gran mayoría enquista esta tradición mesiánica en las generaciones de maestros músicos que más tarde no tendrán otra visión de la música que no pase de la mimesis y de la mecánica instrumental. El acto musical se ha convertido en un artefacto cultural como una entidad propia capaz de objetivizarse, que opera en múltiples niveles y que afecta a muchas personas. Esto hace tambalear la estructura tradicional de la formación profesional en la música y los procesos pedagógicos de la enseñabilidad y de la educabilidad que se dan al interior de éstos.

De acuerdo con los antecedentes teóricos y metodológicos en el desarrollo de la investigación musical y de los procesos de enseñabilidad y educabilidad, se plantea entonces el diseño de un nuevo método de formación musical que conduzca a niveles de creación musical según las condiciones aptitudinales de cada sujeto.

\section{Metodología}

La siguiente investigación se circunscribe a un estudio cuasiexperimental con pre y post test. De esta manera se busca valorar la efectividad del método Cuerpo y oído pensante.

De acuerdo con lo planteado en el marco conceptual, el constructo de interés "creación musical de personas que no han desarrollado su talento musical" está compuesto por tres dimensiones: partitura musical - partitura corporal - partitura textual.

Con un grupo de expertos en lingüística y literatura, artes escénicas, danzas, artes musicales y artes visuales, nos dimos a la tarea de construir una versión preliminar del instrumento de medición, el cual pretende ser aplicado en una muestra piloto, programada para inicios del segundo semestre del 2004, a una población representativa de estudiantes de primer ingreso de la Facultad de Cultura Física Deporte y Recreación de la Universidad Santo Tomás de Colombia. Este proceso se realiza con el fin de establecer evidencias empíricas de su calidad técnica que permitan generar recomendaciones para su mejoramiento.

Se busca construir un instrumento práctico, sencillo de usar y calificar, el cual va acompañado de un instructivo que permita al evaluador, en caso de ser necesario, recurrir al mismo para aclarar dudas con relación a las dimensiones evaluadas.

La partitura textual responde a la definición de una de las dimensiones que permite identificar la coherencia entre el escrito textual, la expresión corporal y la creación musical. Con el fin de que el constructo de interés "creación musical de personas que no han desarrollado su talento musical" pueda objetivarse desde diferentes ópticas, entre ellas la escrita, las cuales permitan observar el proceso creativo en una función integrada de conocimiento sensible, creación artística, desarrollo musical, pensamiento simbólico y pensamiento relacional.

Esta dimensión ha sido construida por un grupo de expertos en lingüística y literatura, que determinaron las categorías que permiten identificar la relación entre el texto textual y el texto musical, en una función integrada de coherencia y de sentido que denote posisionamiento del saber dinamizado (creación musical) a través de su uso (estructura textual).

La partitura corporal responde a la definición de las dimensiones que permiten identificar la coherencia entre la expresión corporal, el escrito textual y la creación musical. Con el fin de que el constructo de interés "creación musical de personas que no han desarrollado su talento musical" pueda objetivarse en lo corporal, a través del movimiento, en una sola función que permita observar el proceso creativo de significación musical y textual que hacen parte del proceso de creación musical de Cuerpo y oído pensante. La ubicación espacio 
temporal y la capacidad de significación de la obra musical a través del cuerpo, permiten la comprensión del proceso de creación musical y su ubicación en un contexto de conocimiento sensible, creación artística, desarroIlo musical, pensamiento simbólico y pensamiento relacional.

Esta dimensión ha sido construida por un grupo de expertos en artes escénicas, artes visuales y danzas, que determinaron los mecanismos para identificar la relación entre la creación musical y el movimiento corporal, en una función integrada de coherencia y de sentido que denote posisionamiento del saber dinamizado (creación musical) a través de su uso (estructura corporal).

La partitura musical responde a la definición de las dimensiones que permiten identificar la coherencia entre la partitura corporal, la partitura textual y la creación musical. Con el fin de que el constructo de interés "creación musical de personas que no han desarrollado su talento musical" pueda objetivarse en estructuras sonoras y rítmicas que permitan valorar el proceso creativo de significación y de apropiación de los elementos para la creación musical.

Esta dimensión ha sido construida por un grupo de expertos en artes musicales y composición que determinaron los mecanismos para identificar la relación entre la creación musical, la creación textual y el movimiento corporal, en una función integrada de coherencia y de sentido que denote posisionamiento del saber dinamizado.

La aplicación piloto pretende ser evaluada por un grupo interdisciplinario de examinadores en cada dimensión. Los cuales deben recibir instrucciones previas en términos de cómo usar el instrumento. Cada prueba se evaluará con tres expertos, con el fin de establecer evidencias de la consistencia entre examinadores.
El constructo de interés "creación musical de personas que no han desarrollado su talento musical" pretende ser calculado como un promedio ponderado de las puntuaciones en las tres dimensiones, en donde la partitura musical tenga el doble de importancia comparado con la partitura textual y corporal.

Para generar los análisis cuantitativos de la calidad técnica del instrumento y para describir el comportamiento de los puntajes en cada una de las tres dimensiones y en el indicador global de creación musical, se usará el paquete estadístico SPSS para Windows.

Con el instrumento depurado se espera proceder a obtener las calificaciones de cada estudiante en las tres dimensiones, para determinar el cociente del total de puntos obtenidos entre el número máximo de puntos posible. El índice de Creación Musical se pretende calcular entonces mediante un promedio ponderado de las tres dimensiones, dando doble ponderación a la partitura musical.

Finalmente, para analizar el grado de consistencia entre jueces, se pretende calcular la correlación entre los puntajes totales otorgados en cada dimensión por los tres examinadores.

\section{Unidades de análisis}

En la consolidación de la estructura conceptual y del diseño del método de formación musical, este primer momento concreta las unidades de análisis en la teoría de las inteligencias múltiples de H. Gardner y la inteligencia musical desarrollada por Xavier Zubiri, respectivamente. Un segundo momento ubica como unidad de análisis los estudiantes que intervienen en el proceso experimental y de control, a partir del pre test y el pos test, como instrumentos de aplicación. Para el tercer momento surgen como unidad de análisis los datos arrojados por la muestra. 


\section{Etapas planteadas en el proceso de diseño de la investigación}

1. Diseño de instrumento "niveles de creación musical"

2. Diseño de instrucciones para los examinadores externos

3. Aplicación piloto

4. Validación del instrumento

5. Aplicación del método

6. Elaboración del documento final.

\section{Discusión}

La creación del método Cuerpo y oído pensante parte de que la educación musical en personas disciplinar y culturalmente no aptas para su práctica debe reconocer en ellas una capacidad natural, biológica, que le permite a través de los sentidos comprender, aprehender y construir estructuras llenas de sentido, solo por la condición biológica que tenemos de escuchar y de comunicar con otros lenguajes. De igual manera, es fundamental abordar el problema de lo social cultural, en el sentido estricto de las interpretaciones, visiones y constructos culturales sociales que se han anidado en la sociedad, con el pretexto de organizar las movilidades sujetales que en muchos casos lo que hacen es cerrar las posibilidades de diálogo entre ellas y provocar en el individuo una serie de inseguridades que le determinan sus campos de acción y que lo excluyen con sus sistemas simbólicos clausurados, sin ni siquiera conceder al individuo la exploración mínima de sus capacidades en otros campos o dominios del conocimiento, en este caso el de la educación musical.

Esta tendencia a la paradigmatización de los saberes y habilidades connaturales del ser humano es lo que ha generado hombres y mujeres que a los cuarenta años de vida se consideran frustrados en diversos campos, como la música, porque alguna vez el sistema mismo los catalogó como insuficientes para su práctica. El método de formación musical de Cuerpo y oído pensante, plantea el reto de romper la estructura tradicional en la que unos pocos, lo dotados, pueden acceder al conocimiento musical, apropiarse de él y hacerlo parte de su vida.

En tercer lugar, el problema de las prácticas y de los supuestos teóricos que condicionan las prácticas es parte constitutiva del método de Cuerpo y oído pensante, ya que las distancias entre lo que dicen las teorías y lo que realmente puede lograr el ser humano a través de los sentidos es realmente enorme. Las prácticas comunes de los seres humanos son posibles a través de la realización de los sentidos. En este caso, el gran problema se centra no en que existan personas con aptitudes y personas que no, sino en que las teorías han delimitado ya de entrada su existencia al ubicar categorías sin opuestos como el ritmo, lo afinado, etc., sin dejar la posibilidad de que en dicho mundo, el de la música, se permee algo que esté por fuera de sus estructura tradicional y convencional.

Es momento de redimensionar las prácticas y de cambiar los sistemas de códigos con los que nos entendemos al interior de la música, abrir los campos conversacionales, validar y reconocer que el otro discurso, nuevas escalas, ritmos... puede llegar a ser igualmente musical y válido. Si hoy en día puede conversar la filosofía con la neurociencia, ipor qué no la música puede dialogar con otro tipo de escalas que no sean las diatónicas o las cromáticas, o con otro tipo de ritmos que no necesariamente necesitan de metrónomos para acomodarse a lo "real"? El último problema es el de la educación, siendo esta la base que nos permite tender el tamiz en donde se puede generar la discusión y la reflexión sobre la operacionalización del método Cuerpo y oído pensante. Las distinciones en la escuela acerca de la educación musical no son más que un indicador de que la formación de quienes dirigen cátedras de música debe reconocer en los sujetos, más que tiempos preestablecidos de rítmicos o arrítmicos, seres sentientes, productivos, e igualmente capaces de 


\section{(92) Universidad Santo Tomás}

operacionalizar sus sentidos para la creación de la música, la composición, el entendimiento de sus códigos, la instrumentalización de las obras que componen y, porque no, el canto y la interpretación.
Las prácticas educativas se deben enfocar al logro de espacios concertados y a la creación de sistemas simbólicos que eliminen distancias comunicacionales anidadas en las disciplinas y enquistadas en las personas.

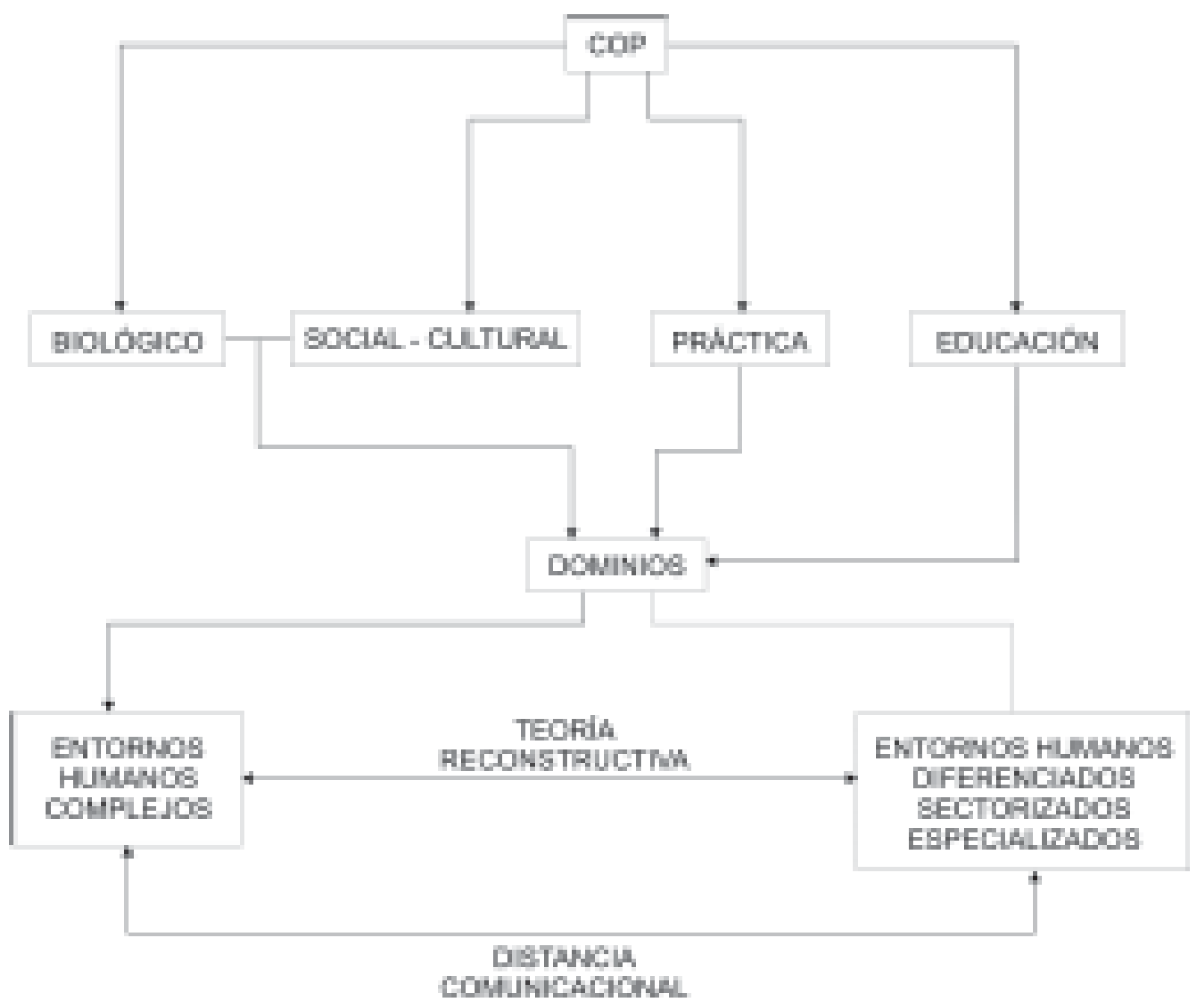

Figura 1. Delimitación de la estructura conceptual de Cuerpo y oído pensante. 


\section{Bibliografía}

Frega, Anna Lucia. 1980. M. M. Vaughan. Creatividad musical, fundamentos y estrategias para su desarrollo. Buenos Aires: División Desarrollo Musical, Casa América.

Elliott. J. 1990. La investigación-acción en educación. Madrid: Morata.

1993. El cambio educativo desde la investigación-acción. Madrid: Morata.

Flórez Ochoa, Rafael. 2000. Hacia una pedagogía del conocimiento. Bogotá: McGraw-Hill.

Fodor, J. 1983. The modularity of Mind. Cambridge. MIT Press.

Fubini, Enrico. 2001. Estética de la música. Madrid: A Machado Libros.

Gadamer, H. Verdad y método.

García Carrasco, J. 2001. Teoría de la educación. Salamanca: Universidad de Salamanca.

Gardner, Howard. 1994. Educación artística y desarrollo humano. Barcelona: Paidós.

Gardner, H. 1994. Estructuras de la mente, la teoría de las inteligencias múltiples. Nueva Cork: Basic books.

Gardner, M. K. 1985. Cognitive psychological to instructional task analysis. In E.

H. Maturana. 1995. La realidad: iobjetiva o construida? Fundamentos biológicos de la realidad. Barcelona: Anthropos.

Hemsy de Gainza, Violeta (editora). 1993. La educación musical frente al futuro. Buenos Aires: Guadalupe.

Hemsy de Gainza, Violeta. 1977. Fundamentos, materiales y técnicas de la educación musical. Buenos Aires: Ricordi Am.

Hemsy de Gainza, V. 1964. La educación musical en el niño. Buenos Aires: Ricordi,.
Hemsy de Gainza, V. 1982. Ocho estudios de psicopedagogía musical. Buenos Aires: Paidós.

Hoffer, C. 1992. "Sociology and Music Education", en: Colwel, R. 1992. Handbook of research on music teaching and learning. USA: Schirmer Books.

Changeux, J. Pierre y Paul Ricoeur. 1999. Lo que nos hace pensar. Barcelona: Península.

J. D. Vincent. 1986. Biologie des passions. Paris: Odile Jacob.

Karmiloff Smith, A. 1994. Más allá de la modularidad. La ciencia cognitiva desde la perspectiva del desarroIlo. Madrid: Alianza.

Swanwick, K. 1994. Musical knowledge: intuition, analysis and music education. London and New York: Routledge.

Swanwick, K. 1997. "Assessing musical quality in the National Curriculum", British Journal of Music Education 14(3).

Madsen, C. K. Yarbrough, C. 1980. Competency based music education. Engelwood Cliffs, N.J.: PrenticeHall Inc.

Madsen, C. K. 1993. Developing prospective music teachers. A festschrift in honor of Helmut Moog. Frankfurt am Main: Peter Lang.

Madsen, C. K., Standley, J. M., \& Cassidy, J. W. 1989. Demonstration and recognition of high/low contrasts in teacher intensity. Journal of Research in Music Education, 37, p.p. 87-94.

R. Murray Schafer. Hacia una educación sonora. Pedagogías Musicales Abiertas. Buenos Aires.

SCHÖN, D. 1992. La formación de profesores reflexivos. Madrid: Paidós/M.E.C.

Zubiri, X. 1984. Inteligencia sentiente. Inteligencia y realidad. Madrid: Alianza. 